\title{
Voreilige Schlüsse, irrationale Entscheidungen
}

Fragestellung: Kommt es bei der Behandlung des Restless-legsSyndroms (RLS) mit dopaminerger Medikation vermehrt zu einer veränderten Entscheidungsfindung?

Hintergrund: Das RLS ist eine recht häufige sensomotorische Störung und oft mit weiteren neuropsychiatrischen Störungen wie Depression und Panikstörung assoziiert. Dopaminerge Substanzen wie Levodopa und Non-Ergot-Dopaminagonisten sind die Medikamente der ersten Wahl. $\alpha$-2-Delta-Liganden wie Gabapentin und Pregabalin sind wirksam, aber in Deutschland nicht für das RLS zugelassen. Die Dopaminagonisten haben jedoch unerwünschte Arzneiwirkungen wie Impulskontrollstörungen oder Augmentation. Mehrere Studien zu den kognitiven Funktionen von RLS-Patienten ergaben keine konsistenten Ergebnisse. Teils wurde eine reduzierte psychomotorische
Heim B, Pertl MT, Stefani A et al. Haste makes waste: Decision making in patients with restless legs syndrome with and without augmentation. PLOS ONE 2017; 12: e0174793. https://doi. org/10.1371/journal. pone. 0174793 Geschwindigkeit und Wortflüssigkeit oder ein reduziertes Entscheidungsverhalten bei mehrdeutigen Stimuli oder Belohnungssuche gefunden, teils waren die Patienten aber auch überdurchschnittlich bei Aufgaben zu
Aufmerksamkeit, Sprachfähigkeiten, Exekutivfunktionen oder Gedächtnis. Kognitive Beeinträchtigungen wurden bisher aber bei RLS-Patienten nicht untersucht. Da es theoretisch Unterschiede gibt zwischen RLS-Patienten mit und ohne Augmentation, wurde diesem Aspekt in der vorliegenden Studie besondere Beachtung zuteil.

Patienten und Methodik: 64 RLS-Patienten ohne Demenz wurden untersucht. Es wurde eine Aufgabe verwendet, die die Anzahl der Informationen beurteilt, bevor eine Versuchsperson eine Entscheidung trifft (Beads Task). Als Vergleichsgruppe dienten 21 gesunde Kontrollen.

Ergebnisse: Es spielte keine Rolle, ob die Patienten eine Augmentation hatten oder nicht. Alle Patienten sammelten aber weniger Informationen vor einer Entscheidung als die Kontrollgruppe. Die Patienten trafen auch mehr irrationale Entscheidungen und hier schnitten die Patienten mit Augmentation besonders schlecht ab.

Schlussfolgerungen: RLS-Patienten neigen $\mathrm{zu}$ vorzeitigen Schlussfolgerungen und entscheiden bei unzureichenden Anhaltspunkten. Patienten mit RLS und Augmentation trafen öfter irrationale Entscheidungen.

\section{- Kommentar von Markus Weih, Nürnberg}

\section{Neuropsychologie des RLS}

Das RLS ist eine häufige und teils schwere Erkrankung. Zwar gibt es viele Patienten, die die Symptome nur intermittierend oder leicht haben und nach Ausschluss symptomatischer Fälle vom Hausarzt behandelt werden können. Die Behandlung ist aber in vielen Fällen kompliziert durch unerwünschte Arzneiwirkungen, Unwirksamkeit oder sogar Verschlechterung unter Therapie - wie bei Augmentation. Hier ist der Facharzt gefragt. Dazu kommt das Problem, dass viele Psychopharmaka, vor allem Dopaminantagonisten ein RLS hervorrufen können. Im idiopathischen Fall muss nicht selten bei Versagen der dopaminergen Therapie bei schweren oder sehr schweren Fällen auf Oxycodon/Naloxon als Second-line umgestellt werden.

Die vorliegende Studie ist klein und hat mit einem Punkt nur einen niedrigen Jadad-Score. Dennoch ist sie wertvoll, da sie zeigte, dass es nicht nur bei Psychosen oder Suchterkrankungen sondern auch bei Erkrankungen mit dopaminerger Überstimulation wie dem RLS zu voreiligen Schlussfolgerungen und irrationalen Entscheidungen kommt, was natürlich bei einer Substanzgruppen wie Dopaminagonisten besonders heikel ist. Voreilige Schlussfolgerungen und irrationale Entscheidungen als Extremvariante normalen Denkens sind die eigentlichen zugrunde liegenden kognitiven Fehler der Impulskontrollstörungen, über die der Patient ja aufgeklärt werden muss. Das verwendete Modell (Beads Task) ist jedoch auch

grundsätzlich interessant, da es uns viel über die menschliche Denk- und Verhaltensweisen sagt und hilft, die neuropsychologische Grundlage von Entscheidung bei limitierter Information beziehungsweise irrationale Entscheidungen zu verstehen. Für die Praxis lässt sich aus der vorliegenden wissenschaftlichen Studie nichts Konkretes ableiten. Das Problem der Augmentation beziehungsweise Impulskontrollstörungen sollte aber nicht vergessen werden. Empfohlen wird das Absetzen der Substanz, die das Problem auslöst, und das Umstellen auf zugelassene Opiate. Gabapentin oder Pregabalin sind nicht zugelassen, stehen aber nach entsprechender Aufklärung in der Praxis durchaus als Alternativen zur Verfügung.

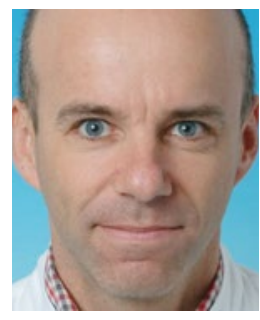

Prof. Dr. med. Markus Weih, Nürnberg

Facharzt für Neurologie, Psychiatrie und Psychotherapie, Nervenärztliche Gemeinschaftspraxis E-Mail:weih@nervenaerzteallersbergerstrasse.de 